\title{
DISTRIBUCIÓN Y SIMILITUD DE LOS PECES DULCEACUÍCOLAS DEL MUNICIPIO GIBARA, HOLGUÍN, CUBA
}

\author{
Enrique Reynaldo, Antonio Vega, Alejandro Fernández y Elier Córdova \\ Centro de Investigaciones y Servicios Ambientales y Tecnológicos de Holguín, Calle $18 \mathrm{~s} / \mathrm{n}$ e/ 1ra. \\ y Maceo. Reparto. El Llano, Holguín, 80100, Cuba. \\ ereynaldo@cisat.cu, vega@cisat.cu, ale@cisat.cu, elier@cisat.cu.
}

\section{RESUMEN}

Las primeras indagaciones en la estructura y composición de la ictiofauna en los ríos de Cuba la realizó el naturalista cubano Felipe Poey. Numerosas han sido las investigaciones realizadas en este campo, principalmente en la región occidental y central de Cuba. Este trabajo se propuso determinar la distribución y similitud de los grupos de especies ícticas dulceacuícolas de las diferentes localidades del municipio Gibara. Se determinó el número de especies por cada localidad, se calculó la similitud a través del índice de Bray-Curtis y el rango de distancia de disimilitud entre las localidades mediante el escalado multidimensional no métrico, el cual permite construir un mapa de ordenamiento del número específico de dimensiones. Se registraron 30 especies, de ellas dos marinas y dos nuevos morfos, que probablemente constituyan especies nuevas. Se agruparon en nueve órdenes, 14 familias y 25 géneros. Las localidades con mayor número de especies fueron las que presentaron una amplia red fluvial tales como, Uñas con 22, Floro Pérez 20, Velasco Uno 19, Cañada de Melones 18, Bocas 17 y Velasco Dos 16. E1 30\% de las especies son endémicas, con un mayor número en las localidades Velasco Uno, Uña, y Velasco Dos. Las especies exóticas estuvieron presentes en un $18.75 \%$, distribuidas en mayor cantidad en Uñas y Cañada de Melones con seis especies cada una. Las especies autóctonas representaron el 50\% del total especies ubicadas principalmente en Floro Pérez, Uña y Velasco Uno. Los resultados del estudio de asociaciones mostraron dos grandes grupos a un $46 \%$ de similitud y se obtuvo un nivel de estrés de 0.01 lo que demuestra un buen ajuste del orden espacial y proporción de la distancia ecológica.

Palabras clave: ecología, peces dulceacuícolas, Gibara.

Title: Composition and distribution of the freshwater fishes of Gibara, Holguin, Cuba.

\section{ABSTRACT}

The first investigations on the structure and composition of fish fauna in the rivers of Cuba was carried out by the Cuban naturalist Felipe Poey. Numerous investigations in this field, mainly in the western and central of Cuba have been conducted. This work aimed to determine the distribution and similarity of groups of freshwater fish species of different localities of the municipality Gibara. The number of species for each locality and the similarity by the test Bray-Curtis and the distance range of dissimilarity between the localities using non-metric multidimensional scaling, allowing to construct a map of ordering a specific number of dimensions. In total 30 fish species were recorded, among them two marine and two morphs new that probably constitute new species. These species were clustered in nine orders, 14 families and 25 genera. The localities with more recorded species were those with wider hydrographic basin web, taking into account identified and named rivers, such as: Uñas with 22, Floro Pérez 20, Velasco Uno 19, Cañada de Melones 18, Boca 17 and Velasco Dos 16. The $30 \%$ recorded species were endemic, with more number in the localities Velasco Uno, Uñas and Velasco Dos. Alien species were $18.75 \%$, obtained in Uñas and Cañada de Melones with six 
species per places well represented. Native species were $50 \%$ all species located primarily in Floro Pérez, Uña and Velasco One. The fish associations among localities showed two big groups with a $46 \%$ similarity and it was obtained stress level of 0.01 which shows a good fit of the spatial order and proportion of ecological distance.

Keywords: ecology, fish freshwater, Gibara.

\section{INTRODUCCIÓN}

Para la región nororiental de Cuba se reportan hasta el momento 26 especies de peces dulceacuícolas, 14 de ellas se consideran estrictamente de agua dulce para un $45.6 \%$ del total consignado para Cuba, con nueve endémicas (39.1\%), valor relativamente bajo en comparación con la ictiofauna endémica cubana (Ponce de León, 2011a). La ictiofauna cubana cuenta con 57 especies que en algún momento de su ciclo vital hacen uso de los ecosistemas de agua dulce (Vales et al., 1998), de las cuales sólo 35 se consideran estrictamente de agua dulce y 23 endémicas (40\%). El grupo mejor representado es el orden Cyprinodontiformes el cual incluye las familias: Cyprinodontidae y Poeciliidae, esta última con 16 especies, de las cuales más del 93\% son endémicas, lo que representa el $65 \%$ del total de las especies endémicas de peces cubanos (Ponce de León y Rodríguez, 2010b). Los principales géneros que conforman este orden son los endémicos Girardinus, Quintana y Cubanichthys (Rosen, 1975).

En la isla no se reportan especies dulceacuícolas primarias, es decir, aquellas que evolucionaron a partir de otras especies también de agua dulce, sino especies secundarias; estas últimas han evolucionado a partir de especies marinas, y son capaces de tolerar varios grados de salinidad (Myers, 1938). En Cuba existen especies marinas habitando en ecosistemas dulceacuícolas que las definen como especies periféricas de procedencia marina, invaden los ríos e incluso llegan a reproducirse en agua dulce (Barus et al., 1998). En estos ecosistemas se puede encontrar especies introducidas con diferentes objetivos, ya sea para el consumo humano o como controladores biológicos (Ponce de León, 2011b).

Los peces de agua dulce, se adaptan mejor en aguas eutróficas y profundas (Ramírez et al., 1998) y en sitios con variedad de hábitats, donde presentan una mayor diversidad de especies y menor grado de dominancia (Machado y Moreno, 1993).

Las principales problemáticas que atentan contra la conservación de los ecosistemas, como de las especies autóctonas dulceacuícolas en Cuba, son la acción sinérgica de múltiples estresores (contaminación, represamiento, reducción de nutrientes, degradación de los hábitats, cambios globales, sobrepesca, etc.). Estos factores son los que han provocado la principal disminución de los peces dulceacuícolas en Cuba. Un peligro aún mayor lo representa la introducción de peces exóticos en acuatorios naturales, ocasionando a la fauna autóctona la extinción y deterioro de su hábitat (Quammen, 1996).

\section{OBJETIVO}

-Determinar la distribución espacial y similitud de los grupos de especies ícticas dulceacuícolas de las diferentes localidades del municipio Gibara, Cuba. 


\section{MATERIALES Y MÉTODOS}

Área de estudio. El municipio Gibara se localiza en la costa norte de Cuba, al noroeste de la provincia Holguín, entre los $21^{\circ} 06^{\prime} 34^{\prime \prime}$ Latitud Norte y los $76^{\circ} 07^{\prime} 54^{\prime \prime}$ Longitud Oeste, con una extensión territorial de $619 \mathrm{~km}^{2}$, ocupa el noveno lugar en superficie que representa el $6,7 \%$ de la superficie total de la provincia de Holguín. El mismo está constituido por las localidades siguientes: Caletones (C), Gibara Uno(G1), Gibara Dos (G2), Floro Pérez(FP), Bocas (B), Velasco Uno (V1), Velasco Dos (V2), Uñas (U), Arroyo Seco (AS) y Cañada de Melones (CM) (Fig. 1).

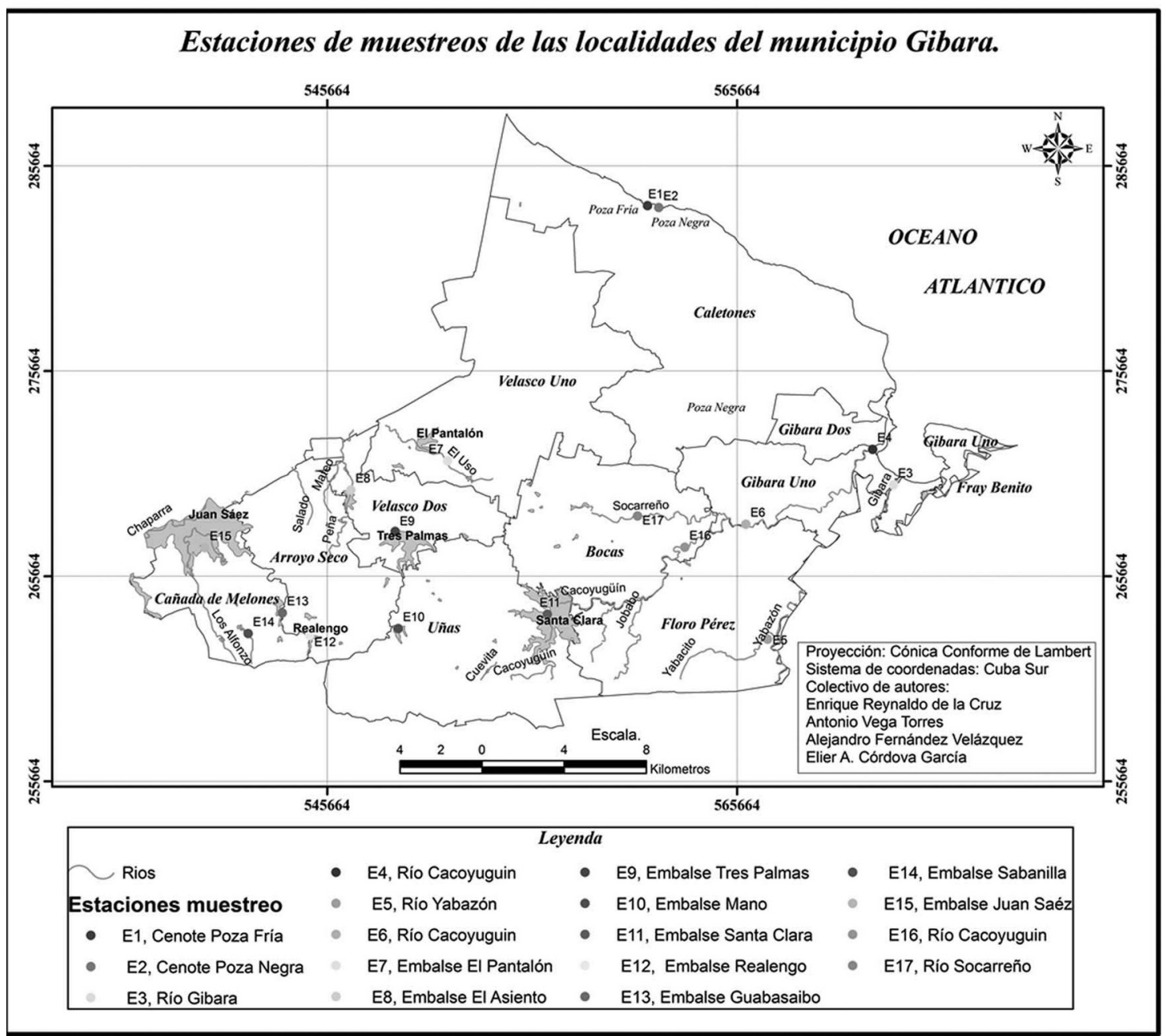

Figura 1. Estaciones de muestreos en las localidades del municipio Gibara.

Diseño de muestreo. Se tomaron 17 estaciones de muestreo con sus coordenadas (Tabla I), ubicadas en correspondencia con cada localidad, tanto en embalses, como en ríos y afluentes principales. El período de muestreo fue realizado en los meses de febrero, marzo, abril, junio y julio del 2014. Se utilizó como referencia el inventario ictiofaunístico dulceacuícola en el norte oriental de Holguín (Fernández y Ocaña, 2011). 
Tabla I. Localidades, sitios muestreados, coordenadas y estaciones en el área de estudio.

\begin{tabular}{|l|l|c|c|c|}
\hline \multirow{2}{*}{ Localidades } & \multicolumn{1}{|c|}{ Sitios muestreados } & \multicolumn{2}{c|}{$\begin{array}{c}\text { Coordenadas planas rectangulares } \\
\text { (Cuba Norte) }\end{array}$} & Estaciones \\
\hline \multirow{2}{*}{ Caletones } & Cenote Poza Fría & 561.310 & 283.779 & E1 \\
\cline { 2 - 5 } & Cenote Poza Negra & 561.827 & 283.635 & E2 \\
\hline Gibara Uno & Río Gibara & 572.242 & 283.635 & E3 \\
\hline Gibara Dos & Río Cacoyuguin & 572.291 & 271.769 & E4 \\
\hline \multirow{3}{*}{ Floro Pérez } & Río Yabazón & 567.102 & 262.625 & E5 \\
\cline { 2 - 5 } & Río Cacoyuguin & 565.871 & 268.215 & E6 \\
\hline Velasco Uno & Embalse El Pantalón & 551.502 & 271.281 & E7 \\
\hline \multirow{3}{*}{ Velasco Dos } & Embalse El Asiento & 546.768 & 269.929 & E8 \\
\cline { 2 - 5 } & Embalse Tres Palmas & 548.977 & 267.855 & E9 \\
\hline \multirow{3}{*}{ Uñas } & Embalse Mano & 549.104 & 263.131 & E10 \\
\cline { 2 - 5 } & Embalse Santa Clara & 556.400 & 263.8319 & E11 \\
\hline \multirow{3}{*}{ Arrollo Seco } & Embalse Realengo & 544.802 & 262.773 & E12 \\
\cline { 2 - 5 } & Embalse Guabasaibo & 543.468 & 263.875 & E13 \\
\hline \multirow{2}{*}{ Cañada de Melones } & Embalse Sabanilla & 541.801 & 262.867 & E14 \\
\cline { 2 - 5 } & Embalse Juan Saéz & 539.696 & 267.049 & E15 \\
\hline & Afluente del Río & 563.099 & 267.078 & E16 \\
\cline { 2 - 5 } & Río Socarreño & 560.801 & E17 \\
\hline
\end{tabular}

Método de muestreo. Se realizó la captura de los peces con un jamo de $3 \mathrm{~mm}$ de luz de malla (45 x 50 x $180 \mathrm{~mm}$ ) y una red de $2 \mathrm{~m}$ de longitud y $1 \mathrm{~m}$ de cuerpo con lastre inferior en cada cuerpo de agua. Los organismos capturados se preservaron en pomos con etanol al $90 \%$ y en formol al $10 \%$, a $4^{\circ} \mathrm{C}$. En el laboratorio, los organismos fueron lavados y luego identificados utilizando literatura especializada: Alayo (1973; 1974) y Ponce de León et al. (2010b). La identificación de la especies se realizó también, usando sus características morfológicas de acuerdo a las descripciones originales de Ariagna et al. (2010).

Análisis de los resultados. Se determinó la cantidad de especies y se tabuló la presencia (1) o ausencia (0) de éstas en todas las localidades. Para analizar la similitud ictiológica entre localidades se calculó el Índice de Bray-Curtis (1957) empleando la transformación presencia-ausencia. Este índice es de gran utilidad para este tipo de estudio, debido a que no se ve afectado por los cambios en las unidades de medida y es uno de los más confiables dentro de los índices de similitud. Además de ser uno de los más utilizados para detectar pequeñas diferencias entre datos ecológicos recogidos en diferentes lugares de muestreo (Michie, 1978). Se utilizó la prueba de escalado multidimensional no métrico. El MDS grafica la distancia basada en coordinar las unidades del estudio, basado en el rango de disimilitud. Se considera el más adecuado para el análisis de los patrones en la estructura de las comunidades, debido a que evita asunciones de linealidad y brinda mapas de precisión de las unidades con un orden espacial y proporción de la distancia ecológica (Clarke y Warwick, 2001). El valor de estrés representa en dos dimensiones las relaciones entre las muestras en el espacio multidimensional. Valores $<0,15$ indican un buen ajuste (Clarke y Warwick, 2001). Se siguió el algoritmo metodológico de Field et al. (1982) y fue usado el programa PRIMER 5 versión 5.2.8 (2001) (Plymouth Routines in Multivariate Ecological Research) para Windows desde: http://www.primer-com. 


\section{RESULTADOS}

Distribución por localidades. En el municipio Gibara fueron registradas 30 especies ictiológicas, de ellas dos marinas que se encontraban habitando en ecosistemas dulceacuícolas y dos nuevos morfos que probablemente constituyan especies nuevas, no incluidos en el total de especies. Estas especies se distribuyeron en nueve órdenes, 14 familias y 25 géneros. El $34 \%$ de las especies registradas fueron endémicas, con un endémico local Lucifuga dentata holguinenses (Díaz-Pérez, Nieto y Abio, 1987), el que ha sido categorizado en Peligro Crítico (González et al., 2012) y fueron registradas seis especies introducidas. Las localidades con mayor número de especies fueron las que presentaron una amplia red fluvial: Uñas, Floro Pérez, Velasco Uno, Cañada de Melones, Boca y Velasco Dos (Fig. 2). Las especies con mayor número de reportes entre las localidades de estudio fueron: Gambusia puncticulata (Poey, 1854), Gambusia punctata (Poey, 1854), Anguilla rostrata (Lesueur, 1817), Limia vittata (Guichenot, 1853), Nandopsis tetracanthus (Valenciennes, 1831), Poecilia reticulata (Peters, 1859), Clarias gariepinus (Burchell, 1822), Micropterus salmoides (Lacépède, 1802), Cyprinus carpio (Linnaeus, 1758), Awaous banana (Lichtenstein, 1822) y Sicydium plumieri (Bloch, 1786). Del total de especies inventariadas, dos fueron periféricas y reportadas solamente para la localidad de Caletones, Megalops atlanticus (Valenciennes, 1847) y Stegastes partitus (Poey, 1868).

Tabla II. Cantidad de especies endémicas en cada localidad del municipio Gibara. Presencia (1) y ausencia (0): Caletones (C), Gibara Uno (G1), Gibara Dos (G2), Floro Pérez (FP), Bocas (B), Velasco Uno (V1), Velasco Dos (V2), Uñas (U), Arroyo Seco (AS) y Cañada de Melones (CM).

\begin{tabular}{|l|c|c|c|c|c|c|c|c|c|c|}
\hline Especies & $\mathrm{C}$ & $\mathrm{G} 1$ & $\mathrm{G} 2$ & $\mathrm{FP}$ & $\mathrm{B}$ & $\mathrm{V} 1$ & $\mathrm{~V} 2$ & $\mathrm{U}$ & $\mathrm{AS}$ & $\mathrm{CM}$ \\
\hline $\begin{array}{l}\text { Cubanichthys cubensis } \\
\text { (Eigenmann, 1903) }\end{array}$ & 0 & 0 & 0 & 1 & 0 & 0 & 0 & 0 & 0 & 0 \\
\hline $\begin{array}{l}\text { Gambusia punctata } \\
\text { (Poey, 1854) }\end{array}$ & 1 & 1 & 1 & 1 & 1 & 1 & 1 & 1 & 1 & 1 \\
\hline $\begin{array}{l}\text { Girardinus denticulatus } \\
\text { (Garman, 1895) }\end{array}$ & 0 & 0 & 0 & 1 & 1 & 1 & 1 & 1 & 1 & 1 \\
\hline $\begin{array}{l}\text { Girardinus metallicus } \\
\text { (Poey, 1854) }\end{array}$ & 0 & 0 & 1 & 1 & 0 & 0 & 0 & 1 & 0 & 0 \\
\hline $\begin{array}{l}\text { Joturus pichardi } \\
\text { (Poey, 1860) }\end{array}$ & 0 & 0 & 0 & 0 & 0 & 1 & 1 & 1 & 1 & 0 \\
\hline $\begin{array}{l}\text { Limia vittata } \\
\text { (Guichenot, 1853) }\end{array}$ & 0 & 1 & 1 & 0 & 1 & 1 & 1 & 0 & 1 & 1 \\
\hline $\begin{array}{l}\text { Lucifuga dentata holguinensis } \\
\text { (Día-Pérez Nieto y Abio, 1987) }\end{array}$ & 1 & 0 & 0 & 0 & 0 & 0 & 0 & 0 & 0 & 0 \\
\hline $\begin{array}{l}\text { Nandopsis ramsdeni } \\
\text { (Howell-Rivero, 1936) }\end{array}$ & 0 & 0 & 0 & 0 & 0 & 1 & 0 & 1 & 0 & 0 \\
\hline $\begin{array}{l}\text { Nandopsis tetracanthus } \\
\text { (Valenciennes, 1831) }\end{array}$ & 1 & 1 & 1 & 0 & 1 & 1 & 1 & 1 & 1 & 1 \\
\hline \begin{tabular}{l} 
Total \\
\hline
\end{tabular} & 3 & 3 & 4 & 4 & 4 & 6 & 5 & 6 & 5 & 4 \\
\hline
\end{tabular}

Especies endémicas. Las localidades con mayor número de endemismo fueron Velasco Uno, Uña y Velasco Dos (Fig. 3). Con relación al endemismo local, fueron identificados dos nuevos morfos que probablemente constituyan especies nuevas y una en peligro de extinción. La única especie endémica presente en todas las localidades fue G. punctata (Poey, 1854), y la exclusiva a una sola localidad fue L. dentata holguinensis (Díaz-Pérez, Nieto y Abio, 1987) ubicada en la Reserva Ecológica de Caletones (Tabla II). 


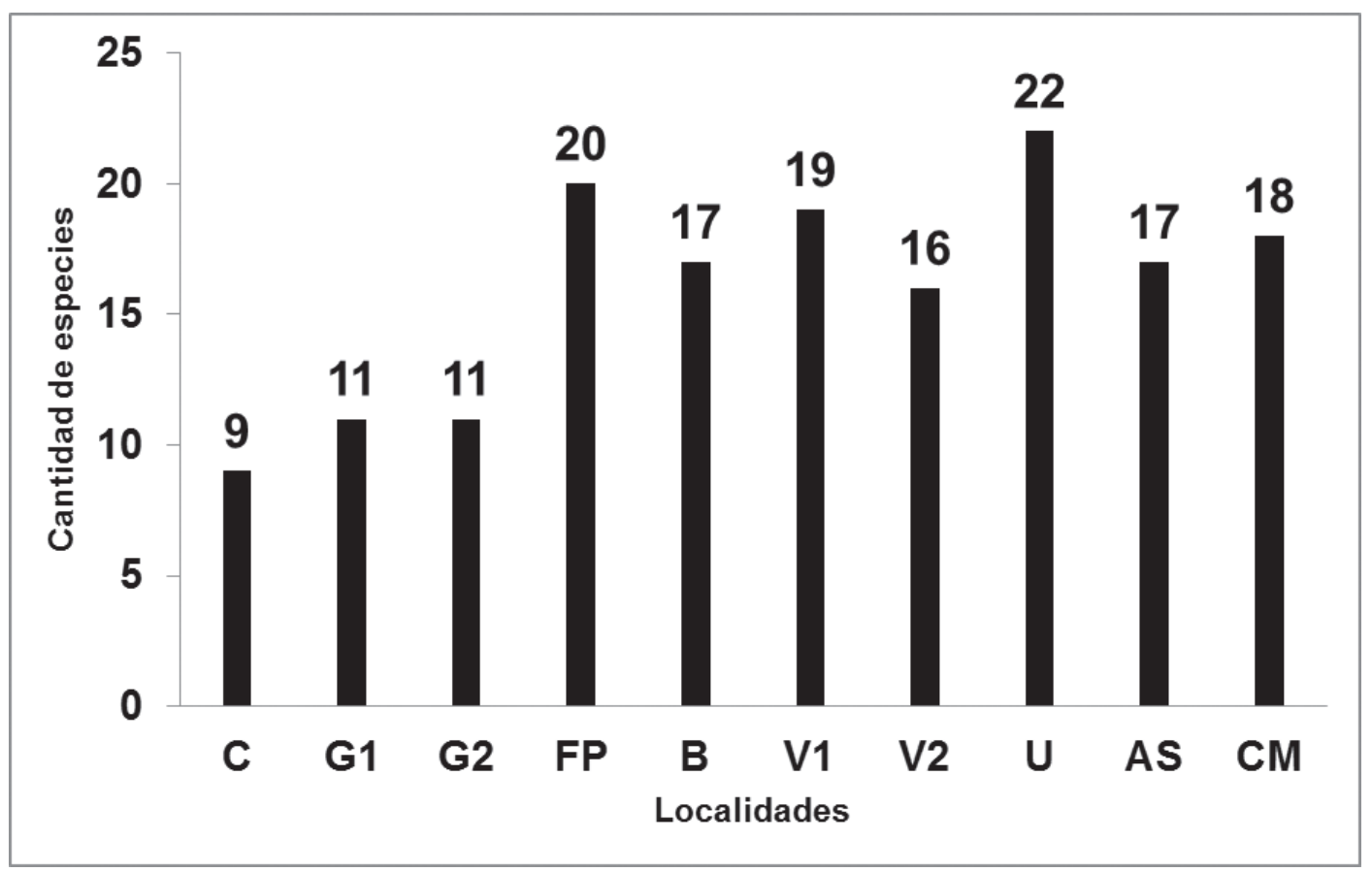

Figura 2. Número de especies por localidades del municipio Gibara.

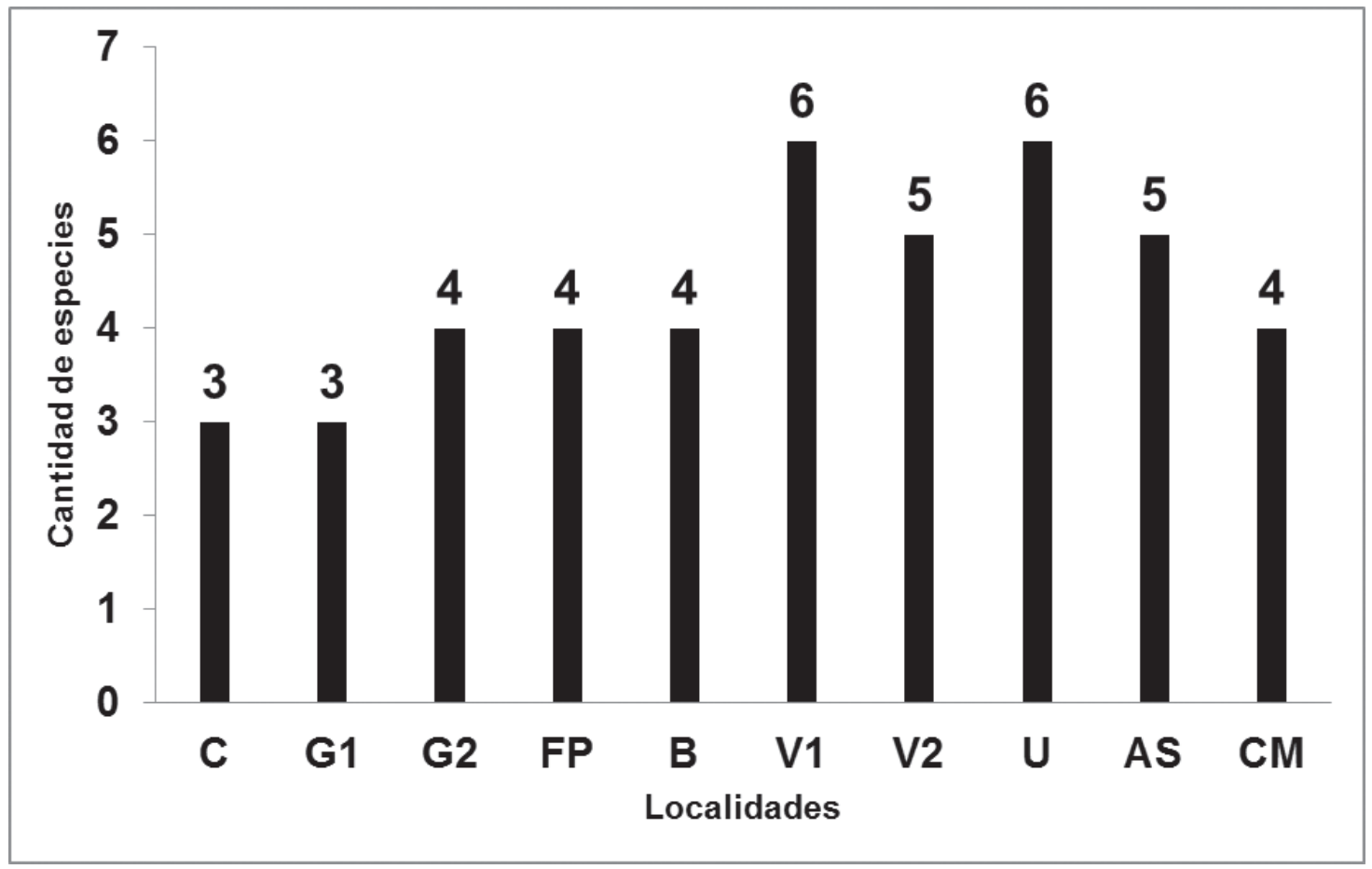

Figura 3. Número de especies endémicas por localidades del municipio Gibara. 
Especies exóticas. Las especies exóticas representaron el 18.75\% de las 30 especies en el listado taxonómico, presentes en 10 localidades del municipio Gibara. Las localidades con mayor cantidad de especies introducidas fueron Uñas y Cañada de Melones con seis especies cada una, y con cinco especies Bocas y Arroyo Seco (Tabla III).

Tabla III. Cantidad de especies exóticas en cada localidad del municipio Gibara. Presencia (1) y ausencia (0): Caletones (C), Gibara Uno (G1), Gibara Dos (G2), Floro Pérez (FP), Bocas (B), Velasco Uno (V1), Velasco Dos (V2), Uñas (U), Arroyo Seco (AS) y Cañada de Melones (CM).

\begin{tabular}{|l|c|c|c|c|c|c|c|c|c|c|}
\hline \multicolumn{1}{|c|}{ Especies } & C & G1 & G2 & FP & B & V1 & V2 & U & AS & CM \\
\hline Poecilia reticulata (Peters, 1859) & 1 & 1 & 1 & 1 & 1 & 1 & 1 & 1 & 1 & 1 \\
\hline Clarias gariepinus (Burchell, 1822) & 0 & 1 & 0 & 1 & 1 & 0 & 1 & 1 & 1 & 1 \\
\hline Cyprinus carpio (Linnaeus, 1758) & 0 & 1 & 1 & 1 & 1 & 1 & 1 & 1 & 1 & 1 \\
\hline Oreochromis mossambicus (Peters, 1852) & 0 & 0 & 0 & 0 & 1 & 1 & 0 & 1 & 1 & 1 \\
\hline Aristichthys nobilis (Richardson, 1845) & 0 & 0 & 0 & 0 & 0 & 0 & 0 & 1 & 0 & 1 \\
\hline Micropterus salmoides (Lacépède, 1802) & 0 & 1 & 1 & 1 & 1 & 1 & 1 & 1 & 1 & 1 \\
\hline Total & 1 & 4 & 3 & 4 & 5 & 4 & 4 & 6 & 5 & 6 \\
\hline
\end{tabular}

Especies autóctonas. Las especies autóctonas representaron el 50\% del total de especies, ubicadas principalmente en Floro Pérez, Uña y Velasco Uno. Siendo Gambusia puncticulata (Poey, 1854), Anguilla rostrata (Lesueur, 1817), Sicydium plumieri (Bloch, 1786), Awaous banana (Lichtenstein, 1822) y Agonostomus monticola (Bancroft, 1834), las especies mejor representadas en las diferentes localidades (Tabla IV).

Tabla IV. Cantidad de especies autóctonas en cada localidad del municipio Gibara. Presencia (1) y ausencia (0): Caletones (C), Gibara Uno (G1), Gibara Dos (G2), Floro Pérez (FP), Bocas (B), Velasco Uno (V1), Velasco Dos (V2), Uñas (U), Arroyo Seco (AS) y Cañada de Melones (CM).

\begin{tabular}{|l|c|c|c|c|c|c|c|c|c|c|}
\hline \multicolumn{1}{|c|}{ Especies } & $\mathrm{C}$ & $\mathrm{G} 1$ & $\mathrm{G} 2$ & $\mathrm{FP}$ & $\mathrm{B}$ & $\mathrm{V} 1$ & $\mathrm{~V} 2$ & $\mathrm{U}$ & $\mathrm{AS}$ & $\mathrm{CM}$ \\
\hline Agonostomus monticola (Bancroft, 1834) & 0 & 0 & 0 & 1 & 0 & 1 & 1 & 1 & 1 & 1 \\
\hline Alepidomus evermanni (Eigenmann, 1903) & 0 & 1 & 0 & 1 & 0 & 1 & 1 & 1 & 0 & 0 \\
\hline Anguilla rostrata (Lesueur, 1817) & 1 & 1 & 1 & 1 & 0 & 1 & 1 & 1 & 1 & 1 \\
\hline Awaous banana (Lichtenstein, 1822) & 1 & 0 & 0 & 1 & 1 & 1 & 1 & 1 & 1 & 1 \\
\hline Cyprinodon variegatus variegatus (Lacepède, 1803) & 0 & 0 & 0 & 1 & 1 & 0 & 0 & 1 & 0 & 0 \\
\hline Dormitator maculatus (Bloch, 1792) & 0 & 0 & 0 & 1 & 1 & 1 & 0 & 0 & 0 & 1 \\
\hline Eleotris pisonis (Gmelin, 1789) & 0 & 0 & 0 & 0 & 0 & 0 & 0 & 1 & 0 & 0 \\
\hline Gambusia puncticulata (Poey, 1854) & 1 & 1 & 1 & 1 & 1 & 1 & 1 & 1 & 1 & 1 \\
\hline Gobiomorus dormitor (Lacepède, 1800) & 0 & 0 & 0 & 1 & 0 & 0 & 0 & 0 & 0 & 1 \\
\hline Guavina guavina (Valenciennes, 1837) & 0 & 0 & 0 & 1 & 1 & 0 & 0 & 1 & 1 & 1 \\
\hline Megalops atlanticus (Valenciennes, 1847) & 1 & 0 & 0 & 0 & 0 & 0 & 0 & 0 & 0 & 0 \\
\hline Ophisternon aenigmaticum (Rosen y Greenwood, 1976) & 0 & 0 & 0 & 1 & 1 & 1 & 1 & 0 & 0 & 1 \\
\hline Sicydium plumieri (Bloch, 1786) & 1 & 1 & 1 & 1 & 1 & 1 & 1 & 1 & 1 & 0 \\
\hline Stegastes partitus (Poey, 1868) & 1 & 0 & 0 & 0 & 0 & 0 & 0 & 0 & 0 & 0 \\
\hline Synbranchus marmoratus (Bloch, 1795) & 0 & 0 & 1 & 1 & 1 & 1 & 0 & 1 & 1 & 0 \\
\hline Totales & 6 & 4 & 4 & 12 & 8 & 9 & 7 & 10 & 7 & 8 \\
\hline
\end{tabular}


Similitud de la ictiofauna por localidades. Las asociaciones de peces en Gibara mostraron dos grandes grupos a un $46 \%$ de similitud (Fig. 4), el primero incluye solo a Caletones y en el segundo grupo las restantes localidades. En el segundo grupo se observó otros dos subgrupos a un $71 \%$ de similitud, el primero constituido por Gibara Uno y Gibara Dos, ambos después de Caletones con el menor número de especies y el segundo subgrupo constituido por el resto de las localidades con mayor número de especies. El porciento de similitud para el primer subgrupo Gibara Uno y Gibara Dos fue de $81 \%$, diferente al segundo subgrupo que presentó un porciento de similitud del 76\% (Fig. 4). Sin embargo los porcientos de similitud más alto fue entre Boca y Velasco Dos 97\%, Arrollo Seco y Boca 94\%. Se obtuvo un nivel de estrés de 0.01 (Fig. 5) esto indica un buen ajuste del orden espacial, separándose Caletones de las demás localidades.

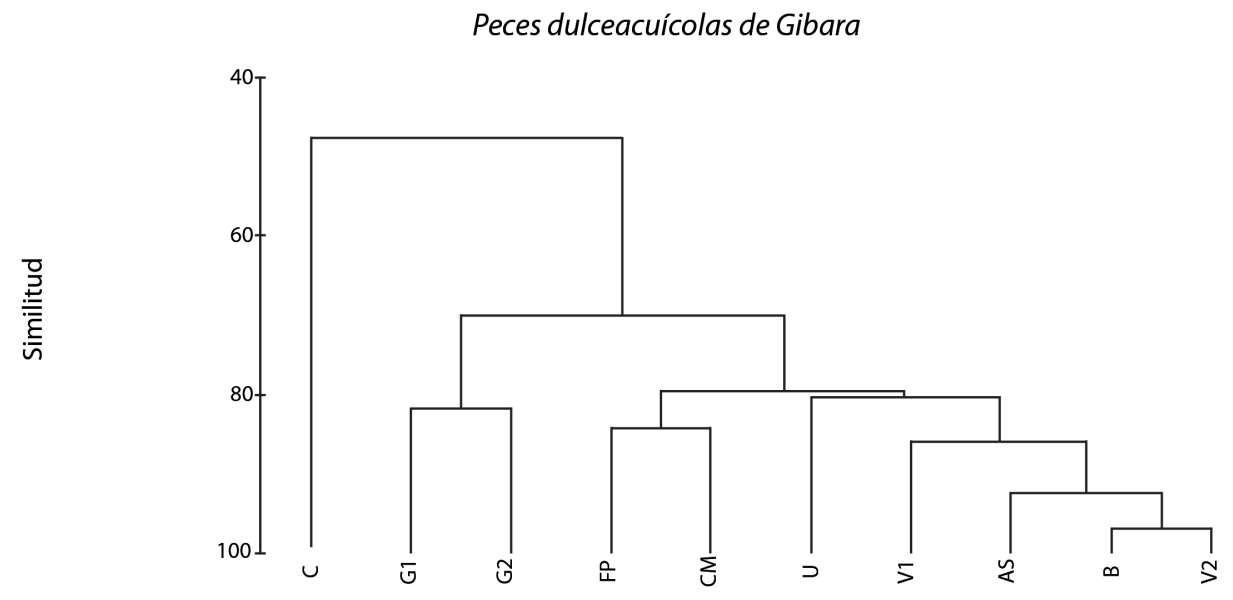

Figura 4. Dendrograma del Índice de Similitud Bray-Curtis de los peces dulceacuícolas del municipio de Gibara. Caletones (C), Gibara Uno (G1), Gibara Dos (G2), Floro Pérez (FP), Bocas (B), Velasco Uno (V1), Velasco Dos (V2), Uñas (U), Arroyo Seco (AS) y Cañada de Melones (CM).

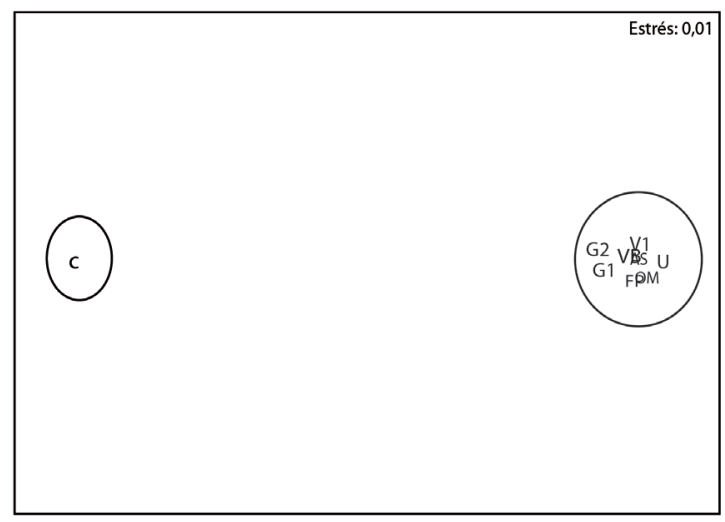

Figura 5. Escalado multidimensional no métrico MSD de los peces dulceacuícolas del municipio de Gibara. Caletones (C), Gibara Uno (G1), Gibara Dos (G2), Floro Pérez (FP), Bocas (B), Velasco Uno (V1), Velasco Dos (V2), Uñas (U), Arroyo Seco (AS) y Cañada de Melones (CM). 


\section{DISCUSIÓN}

Distribución por localidades. Es importante destacar la presencia de peces marinos que llegan habitar ecosistemas dulceacuícolas, como M. atlanticus (Valenciennes, 1847) y S. partitus (Poey, 1868), ambas presentes dentro del sistema espeleolacustre de Caletones. Estas especies son provenientes de ecosistemas costeros y se han adaptado habitar aguas salobres (Wilkens, 1982). Esto permite en las dolinas inundadas de esta región una fauna más diversa. También se evidencia un bajo aislamiento de las especies que habitan en esta dolina y buen estado de la conectividad biológica entre mar-dolina a diferencia de aquellas dolinas más alejadas de la costa.

Con relación a la especie $A$. rostrata (Lesueur, 1817), constituye un taxón que está relacionado filogenéticamente con los táxones centroamericanos, es una especie dulceacuícola secundaria que colonizó con posterioridad las aguas dulces. Constituyendo una especie eurihalina en diversos grados. Esta característica le permite tolerar un alto rango de salinidad en determinados ecosistemas (Rosen, 1975). Gambusia punctata (Poey, 1854) y G. punticulata (Poey, 1854) son las especies que están mejor representadas en Cuba (Ponce de León, 2011a). Poecilia constituye un género que no se ha diversificado en Cuba, debido a la presencia de Girardinus, género endémico, que por exclusión competitiva, ha forzado a Poecilia reticulata (Peters, 1859), a especializarse en una alimentación limnívora (Ponce de León, 2011a).

Limia vittata (Guichenot, 1853), especie de pequeño tamaño y especializada a diferentes condiciones del medio ambiente. Es un pez limnófago y epibentónico, estas características le permiten una creciente adaptabilidad a determinados recursos y condiciones ambientales, posibilitando aumentar la extensión de su área de distribución (Vergara, 1992). Nandopsis tetracanthus (Valenciennes, 1831), presenta una marcada variabilidad de frecuencias fenotípicas. Dentro de una misma población pueden encontrase individuos de diferentes proporciones y coloración. La abundancia de esta especie se encuentra fuertemente correlacionada con la disponibilidad de alimento, el cual limita su densidad, siendo uno de los parámetros más críticos de su nicho ecológico. Presenta genotipos adaptados a condiciones ambientales específicas, limitando su distribución (Vergara, 1992).

Clarias gariepinus (Burchell, 1822), Micropterus salmoides (Lacépède, 1802) y Cyprinus carpio (Linnaeus, 1758), son especies introducidas por el hombre por razones económicas. Estas especies tienen características ecológicas diferentes a las especies nativas de Cuba, producto de su historia evolutiva, lo que determina su éxito o fracaso adaptativo en diferentes ecosistemas. Interactúan con las especies nativas como depredadores o al establecer relaciones de competencia por los recursos del hábitat. Presentan determinadas adaptaciones reproductoras que limitan el éxito de las especies nativas y en algunos casos llegan a convertirse en verdaderas plagas, es el caso de C. gariepinus (Burchell, 1822) (Ponce de León y Rodríguez, 2010b).

Especies endémicas. Caletones es el sitio donde existe altas probabilidades de encontrar nuevas especies y éstas podrían ser evaluadas en alguna de las categorías de amenazas según criterios de la UICN, por el hecho de evolucionar en ecosistemas restringidos. Estas especies que habitan en estos ecosistemas se ven obligadas a especializarse y divergen genéticamente, esto trae como resultado la generación de nuevas especies. Es por esta razón que se debe prestar un especial interés para estudios futuros en el sistema espeleolacustre de Caletones, el más grande de la Isla de Cuba (Panos, 1988).

Lucifuga dentata holguinenses (Díaz-Pérez, Nieto y Abio, 1987), constituye la única especie ictiológica endémica local y categorizada en peligro crítico (UICN, 2011) presente en la Reserva Ecológica de Caletones (García et al., 2011). Es la única especie, dentro de las conocidas para 
el género Lucifuga que fue nombrada con una categoría taxonómica infraespecífica, por lo que no se le reconoce como válido. Sin embargo, datos actuales obtenidos mediante el análisis de caracteres, moleculares y morfológicos, número de radios en la aleta caudal, presencia de ojos desarrollados y ausencia de dientes en los huesos palatinos, entre otras características, claramente indican que se trata de un linaje muy divergente con respecto a la subespecie $L$. dentata dentata (Díaz-Pérez, Nieto y Abio, 1987). Constituye una especie longeva, con un promedio de vida de 80 años aproximadamente y una baja natalidad. Estas características la convierten en una especie muy vulnerable (García et al., 2011).

Vulnerabilidad acentuada, debido al deterioro del hábitat por la actividad agrícola y pastoril como una práctica común (González et al., 2008), el vertimiento de sustancias contaminantes al cuerpo de agua y las constantes modificaciones al ecosistema. Estos mismos factores de amenazas, entre otros tales como alteraciones de la vegetación circundante para facilitar el acceso al espejo de agua, y el impacto del uso frecuente y continuo del cuerpo de agua con fines extractivos o de recreo fueron señalados por García et al. (2011). Entre las especies endémicas mejor distribuidas por su presencia se encuentran G. punctata (Poey, 1854) y N. tetracanthus (Valenciennes, 1831; Tabla II); ambas especies adaptadas a las condiciones ambientales de la región norte oriental de Holguín, coincidiendo con las referidas por Ponce de León y Rodríguez (2010a).

Limia vittata (Guichenot, 1853). Especie endémica presente en ocho localidades del municipio de Gibara. Esta especie se encuentra ampliamente distribuida por toda Cuba incluso en la Isla de Juventud. Su dieta principal se compone de detrito e invertebrados, prefiere zonas bajas con fondo fangoso y abundante vegetación y es tolerante a la salinidad (Ponce de León, 2011a). Es un importante controlador biológico natural al consumir grandes cantidades de mosquitos y larvas, (Rivero, 1951; García y Koldenkova, 1990).

Especies exóticas. La localidad con menor número de especies introducidas fue Caletones debido a su difícil acceso, por el hecho de que carece de red fluvial superficial, sin embargo es notable en el sistema espeleolacustre, un importante flujo subterráneo de agua dulce y su mezcla con el agua de mar, encontrándose alta variabilidad salina espacio temporal, siendo $P$. reticulata (Peters, 1859), una especie capaz de tolerar altas concentraciones de salinidad e introducida como agente biológico controlador de mosquitos (Ponce de León y Rodríguez, 2012). Esta especie a su vez, se convierte en un competidor muy fuerte por el alimento con respecto a las especies autóctonas del lugar, por lo que pudiera constituir un problema para la conservación de los ecosistemas, desplazando a especies nativas tolerantes a la salinidad, tales como Gambusia punctata (Ponce de León y Rodríguez, 2010a).

En Cuba, con excepción de algunas especies como A. rostrata (Lesueur, 1817), N. ramsdeni (Fowler, 1938) y N. tetracanthus (Valenciennes, 1831), no existen peces fluviales nativos de uso comercial para la alimentación humana, esto ha inducido la introducción en la isla de especies exóticas continentales con este fin. Muchas de estas especies están actualmente establecidas en aguas cubanas, entre ellas se encuentran la tilapia Oreochromis aureus (Steindachner, 1864) y O. mossambicus (Peters, 1852), de origen africano, estas han sido introducidas en diferentes partes del mundo, principalmente en el trópico (Lovshin y Popma, 1995). Se han introducido para su cultivo en embalses, sin embargo en muchos lugares han escapado de los centros de acuicultura y cultivos familiares, prosperando en ríos, presas y otros acuatorios, donde compiten por los recursos con muchas especies autóctonas dada su resistencia, voracidad y elevada tasa de crecimiento (Rodríguez et al., 1993). 
Otras especies introducidas para el consumo humano en Cuba son Micropterus salmoides (Lacépède, 1802), originaria de Norteamérica, su expansión artificial en nuevos ecosistemas ha causado serios problemas de supervivencia a otras especies ictiológicas dulceacuícolas (Ponce y Rodríguez, 2010b); es un depredador por excelencia, el cual ha mermado considerablemente a las especies fitófagas Lepomis macrochirus (Rafinesque, 1819), Hypostomus plecostomus (Linnaeus, 1758) y N. tetracanthus (Valenciennes, 1831). En el caso de L. macrochirus (Rafinesque, 1819) (Pez Sol), introducido desde E.U.A, es una especie carnívora que altera la distribución y abundancia de los peces nativos al igual que Ctenopharyngodon idella (Valenciennes, 1844), de origen asiático, ambas especies no se reproducen de forma natural en Cuba. De la familia Poiciliidae han sido introducida algunas especies, como controladores biológicos de mosquitos hematófagos, que trasmiten enfermedades al hombre, entre ellas se encuentra Poecilia sphenop (Valenciennes, 1846), Xiphophorus helleri (Heckel, 1848) y con una mayor distribución $P$. reticulata, (Peters, 1859), esta especie se distribuye principalmente en ríos y embalses naturales o artificiales, su impacto a los ecosistemas naturales no ha sido estudiado en la actualidad (Ponce de León, 2011b).

Clarias gariepinus (Burchell, 1822), especie exótica que estuvo presente en la mayoría de las localidades. Estos peces son capaces de explotar diferentes recursos tróficos a lo largo de su vida (Yalçin et al., 2001a). Presentando un alto potencial reproductivo (Yalçin et al., 2001b) y crecimiento rápido. Se alimenta principalmente de insectos, aunque incluyen otros invertebrados acuáticos en su dieta, al igual que algunos peces endémicos cubanos como Gambusia punctata (Poey, 1854) y Nandopsis tetracanthus (Valenciennes, 1831) (Ponce de León y Rodríguez, 2008). Se ha notado una disminución marcada de las especies nativas en Cuba, después de su introducción. Por lo que resulta importante medir su efecto negativo sobre las poblaciones autóctonos, e implementar estrategias para la disminución de su población (Mugica et al., 2006).

Cyprinus carpio (Linnaeus, 1758), de origen asiático, fue introducida al país en la década del '70 aproximadamente, en lagunas y estanques; desde donde se dispersó hacia diversos ambientes acuáticos. Habita en la cuenca de los ríos que desaguan en los mares, son especies que se adaptan a todos los climas, desde templados hasta tropicales, pero siempre prefieren las aguas tranquilas, estancadas y de poca profundidad (Zambrano y Hinojosa, 1999). No toleran altas salinidades, en ocasiones, cuando viven en la desembocadura de los ríos, crean formas anádromas que incursionan por el mar y al momento de la reproducción nadan río arriba. $\mathrm{Su}$ alimentación principal es crustáceos, moluscos, gusanos, larvas de insectos, plantas y hasta peces pequeños. Es una especie de alta fecundidad y tolerantes a factores ambientales desfavorables (Saikia y Das, 2009).

Especies autóctonas. G. puncticulata (Poey, 1854) fue la especie autóctona mejor representada en todos los sitios de muestreo. Es una especie antillana muy abundante en la mayoría de los cuerpos de agua cubanos. Presenta gran tolerancia a la salinidad y se alimenta de insectos y larvas (Fong y Garcés, 1997), aunque también incluye material vegetal en su dieta (Poey, 1854). Es abundante en estuarios y zonas cercanas al mar y altamente tolerante a la contaminación (Ponce de León y Rodríguez, 2010b). A. rostrata (Lesueur, 1817), hábitat principalmente en arroyos, lagos fangosos, ríos con flujo continuo y bien oxigenados. Se alimenta de insectos y de otros peces de la familia Percidae, Cyprinidae, Ictaluridae y Anguillidae. Es una especie que se desplaza al mar durante su período reproductivo (Page y Burr, 1991). Poco se sabe sobre su hábitat de reproducción y desove, se piensa que sean zonas marinas con temperaturas elevadas y alta salinidad (MacGregor et al., 2008). 
Sicydium plumieri (Bloch, 1786) se registró en casi todas las localidades exceptuando Cañada de Melones. Especie que prefiere habitar en ríos cercanos a zonas montañosas y en estadio juvenil migra al mar. Como la mayoría de los gobios, sus aletas ventrales están unidas para formar un disco de succión. Este disco les permite aferrarse a las rocas en aguas rápidas. Se alimenta principalmente de insectos y algas. Es capaz de cambiar su coloración para que coincida con el fondo como mecanismo de defensa (Kullander, 2003). Awaous banana (Lichtenstein, 1822) especie que hábitat en ríos de aguas claras y arroyos, aunque en ocasiones puede encontrase en zonas fangosas y aguas turbias. Su principal alimento lo constituye las algas filamentosas y detrito, llegando a consumir larvas de insectos cuando las algas filamentosas no están disponibles (Watson, 1996). Es capaz de habitar en aguas salobres en su estadio juvenil, siendo intolerable a la salinidad en estadio adulto. Especie que prefiere vivir en aguas poco profunda donde la temperatura se encuentre entre los $26-28^{\circ} \mathrm{C}$ (Contreras et al., 2002).

Agonostomus monticola (Bancroft, 1834) es otra de las especies autóctonas que mayor registró tuvo en las diferentes localidades. Es una especie que no solo se encuentra en agua dulce, también puede ser localizado en agua salobre y marina en donde realiza su reproducción (Ditty y Shaw, 1996). Presenta un notable interés por ríos con fuertes corrientes, aguas claras y zonas pedregosas, que complementan con la zona de desembocadura hacia el mar, en franjas estuarinas, que soportan de forma indirecta su condición migratoria (Garzón y Wedler, 1997). Es considerado un generalista por comer algas filamentosas y principalmente insectos acuáticos; detritos, caracoles, plantas, moluscos y peces pequeños (Torres-Navarro y Lyons, 1999). Esta especie es capaz de incursionar en aguas marinas en escases de recursos alimenticios, además de ser una especie muy susceptible a los cambios ambientales extremos en el medio (Vergara, 1992).

Similitud de la ictiofauna por localidades. La baja similitud entre Caletones con el resto de las localidades (Fig. 4) podría estar dada a las características peculiares de la región, por ser una zona donde los ecosistemas dulceacuícolas obtienen el agua de forma pluvial y no fluvial como el resto de las localidades, dado al gran desarrollo del karso (Panos, 1988). Hidrológicamente Caletones se encuentra en la parte norte de la zona de estudio, la red fluvial (corrientes superficiales) es prácticamente nula, dado a la existencia del sistema kárstico. Este fenómeno capta la escorrentía superficial y la conduce directamente hacia el acuífero. Otras de las razones de la baja similitud de Caletones con resto de las localidades están dadas al bajo grado de antropización, ya que la misma constituye una Reserva Ecológico, a diferencia de las restantes localidades donde la mayoría de los ríos están represados y existe una acentuada actividad agrícola. Otro aspecto que influye en la baja similitud, lo constituye la diferencia de los diferentes elementos químicos del agua. En Caletones el agua presenta una temperatura más baja, $\mathrm{pH}$ básico y la concentración de oxígeno disuelto es más elevada con relación al agua presente en las restantes localidades (Vergara, 1992).

En la Reserva Ecológica de Caletones el sistema acuífero, presenta una comunicación directa con las aguas marinas, de esta forma las propiedades físico-químicas del agua divergen en relación con el agua dulce de las demás localidades, posibilitando la presencia de una ictiofauna especializada. Gibara Uno y Dos presentaron un nivel de similitud de un $81 \%$, debido a que en ambas localidades el principal afluente de agua dulce proviene del río Gibara y afluentes del río Yabazón. La cuenca fluvial del Río Gibara está sometido al represamiento, la influencia de las poblaciones adyacentes a sus márgenes y la presencia en su ribera de residuales agrícolas.

Las localidades Floro Pérez y Cañada de Melones presentaron un 84\% de similitud, en esta área se encuentran grandes represamientos de agua producto del desarrollo agrícola. Encontrándose ambos en la parte sur de la zona de estudio, con una red fluvial de buen desarrollo. 
Hidrológicamente presentan la cuenca hidrográfica del río Cacoyugüín, la cual es la que ocupa mayor área territorial con sus afluentes: Socarreño, Uñitas, Santa Clara, Cuevitas, Managuaco, Pedregoso y Jobabo. Las restantes localidades se distribuyeron en un pequeño subgrupo presentando una similitud elevada, Boca y Velasco Dos 97\%, Arrollo Seco y Boca 94\%, producto al mayor desarrollo de la red fluvial. Además de la similar incidencia de las precipitaciones producto a su cercanía geográfica, lo que posibilita una mayor homogeneidad en cuanto a la diversidad de especies ictiológicas.

\section{CONCLUSIÓN}

Se determinó un total de 30 especies dulceacuícolas y dos marinas en el municipio de Gibara, encontrándose mayor número de especies en las localidades de Uña y Floro Pérez con 22 y 20 especies, respectivamente. La similitud más baja (en un 46\%) fue entre Caletones con el resto de las localidades y la más alta entre Boca y Velazco Dos con un 97\%. Se obtuvo un nivel de estrés de 0.01 lo que demuestra un buen ajuste del orden espacial y proporción de la distancia ecológica.

\section{AGRADECIMIENTOS}

Este estudio fue desarrollado en el marco del proyecto de Investigación: Evaluación de los riesgos de la diversidad biológica en ecosistemas clave de los municipios Mayarí, Gibara y Moa, en la provincia de Holguín.

\section{LITERATURA CITADA}

Alayo, P. 1973. Lista de los peces fluviales de Cuba. Torreia, 29: 19 -73.

Alayo, P. 1974. Guía elemental de las aguas dulces de Cuba. Torreia, 37: 66-68.

Ariagna, F. L., J. L. Ponce de León, R. S. Rodríguez, D. C. Casane, G. L. Bernatchez y E. M. García. 2010. DNA barcoding of Cuban freshwater fishes: evidence for cryptic species and taxonomic conflicts. Molecular Ecology Resources, 10: 421-430.

Barus, V. M., P. Peñáz y M. T. Prokes. 1998. Some new data on Girardinus cubensis (Poeciliidae) from Cuba. Folia Zoologica, 47 (4): 287-293.

Bray, J. R y J. T. Curtis. 1957. An ordination of the upland forest communities of Southern Wisconsin. Ecological Monographs, 27: 325-349.

Clarke, K. R. y R. M. Warwick. 2001. Change in Marine Communities: An Approach to Statistical Analysis and Interpretation. $2^{\text {nd }}$ edition. PRIMER-E. Plymouth U.K. 172 pp.

Contreras B. S., R. J. Edwards, M. D. Loranzo V. y M. E. García Ramírez. 2002. Fish biodiversity changes in the Lower Rio Grande/Rio Bravo, 1953-1996 a review. Reviews in Fish Biology and Fisheries, 12 (2): 219-240.

Ditty, J. G y R .F. Shaw. 1996. Spatial and temporal distribution of larval striped mullet (Mugilcephalus) and white mullet (M. curema, Family: Mugilidae) in the Northern gulf of Mexico, with notes on mountain mullet, Agonostomus monticola. Bull. Mar. Sci., 59: 271-288. 
Fernández, V. A. y B. F. Ocaña. 2011. Biodiversidad de la zona norte oriental de Cuba: Las Tunas-Holguín y Guantánamo, Informe de Proyecto Nacional de Ciencia y Técnica. Fondos informativos del CISAT-Holguín y GEPROG-AMA-CITMA, 130 pp.

Field, J. G., K. R. Clarke y R. M. Warwick. 1982. A practical strategy for analyzing multispecies distribution pattern. Marine Ecology Progress, 8: 37-52.

Fong, A. G. y G. G. Garcés. 1997. Notas sobre la alimentación de Gambusia puncticulata, Poey (Cyprinodontiformes: Poeciliidae) en un hábitat marino. Biodiversidad de Cuba Oriental, II: $54-58 \mathrm{pp}$.

García, E. M., D. E. Hernández, A. García, A. Chevalier, M. P. Metcalfe, C. L. Bernatchez y D. Casane. 2011. Molecular phylogeny and phylogeography of the Cuban cave-fishes of the genus Lucifuga: Evidence for cryptic allopatric diversity. Molecular Phylogenetics and Evolution, 61: 470-483.

García, I. y L. Koldenkova. 1990. Clave pictórica para las principales especies de peces larvívoros de Cuba. Instituto de Medicina Tropical Pedro Kourí Cuba, 1-55 pp.

Garzón, J. y E. Wedler. 1997. Primer registro del besote Joturus pichardi Poey 1860 (Pisces: Mugilidae) para Colombia y América del Sur. Boletín Ecotrópica, 31: 1-9.

González, P. A., S. Suarez, I. Peña, C. Fernández, A. Lafita, O. y S. Sigarreta. 2008. Estudio comparativo de los matorrales en seis conucos abandonados y del bosque Siempreverde micrófilo en Caletones, Gibara, Cuba. RIACRE, 3 (3): 2-5.

González, A. H., S. L. Rodríguez y I. R. García. 2012. Libro Rojo de los Vertebrados de Cuba. Editorial Academia. La Habana, 304pp.

Kullander, S.O. 2003. Gobiidae (Gobies). In R.E. Reis, S.O. Kullander and C.J. Ferraris, Jr. (eds.) Checklist of the freshwater fishes of South and Central America. Porto Alegre: EDIPUCRS, Brasil, 657-665 pp.

Lovshin, L. L y T. J. Popma. 1995. Commercial production of tilapia fry and fingerlings. Situación del cultivo de la tilapia en Panamá. Memorias del I Simposium Centroamericano sobre cultivo de tilapia, Ed. PRADEPESCA. INCOPESCA. ACUACORPORACION y Universidad Nacional Heredia Costa Rica, 5: 95 -101.

Machado, A, R y H. I. Moreno. 1993. Estudio de la comunidad de peces del río Orituco. Estado Guárico Venezuela, Parte I. Inventario, abundancia relativa y diversidad. Acta Biológica Venezuelica, 14 (4): 77-94.

MacGregor, R. B., A. P. Mathers, J. M. Thompson, J. M. Casselman, S. Dettmers, T. C. LaPan y W.A. Allen. 2008. Declines of American Eel in North America: Complexities associated with bi-national management. In International governance of fisheries ecosystems: learning from the past, finding solutions for the future. Edited by M. G. Schechter, W. W. Taylor and N. J. Leonard. American Fisheries Society, Bethesda 357-381pp.

Michie, M. G. 1978. Data manipulation in cluster analysis and the concept of zero presence. Jour. Math. Geol., 10 (4): 335-345.

Myers, S. 1938. Fresh-water fishes and West Indian Zoogeography. Annual Report of the Board of Regents of the Smithsonian Institution, 92: 339-364. 
Mugica, L., M. Acosta, D. Denis y A. Jiménez. 2006. Disponibilidad de presas para las aves acuáticas en los campos inundados de la arrocera del Sur del Jíbaro durante el ciclo de cultivo del arroz. Journal of Caribbean Ornithology, 19: 97-103.

Page, L. M y B. M. Burr. 1991. A field guide to freshwater fishes of North America north of Mexico. Houghton Mifflin Company. Boston, 432 pp.

Panos, V. 1988. Carsos de Cuba oriental. : Studia Geographica 91. Ed. Geograficky ústav, Brno, Checoslovaquia, 194 pp.

Ponce de León, J. L. 2011a. La ictiología de aguas dulces. Línea de investigación en la Facultad de Biología de la Universidad de la Habana. Boletín de la Sociedad Cubana de Zoología, 1: 19-25.

Ponce de León, J. L. 2011b. Peces cubanos amenazados de extinción. En: Rostros amenazados de Cuba. Ediciones Polymita. La Habana 1: 224-225.

Ponce de León, J. L y R. S. Rodríguez. 2008. Spatial distribution of freshwater fish in an intermittent Cuban stream. Biología, 22 (1): 31-50.

Ponce de León, J. L. y R. S. Rodríguez. 2010a. Ecology of Cuban species of the family Poeciliidae (Teleostei: Cyprinodontiformes). En Uribe, MC, HJ Grier (eds.). Viviparous fishes II. Florida, New Life Publ., 5: 13-26.

Ponce de León, J. L. y R. S. Rodríguez. 2010b. Peces cubanos de familia Poeciliidae. Guía de campo. Editorial Academia. La Habana. 38 pp.

Ponce de León, J. L. y R. S Rodríguez. 2012. Riqueza y Abundancia relativa de especies de peces de agua dulce en dos localidades de la Isla de la Juventud al final de época de seca de 2008, Revista Cubana de Ciencias Biológicas, 1 (1): 82-84.

Poey, F. 1854. Los guajacones, pececillos de agua dulce. Memorias de la Historia Natural de la Isla de Cuba, 1 (32): 374-390.

Quammen, D. 1996. The song of the Dodo: island biogeography in an age of extinction, Scriber, $702 \mathrm{pp}$.

Ramírez, G. D., G. F. Alberto y V. Y. Viña. 1998. Limnología colombiana. Aportes a su conocimiento y estadísticas de análisis. Editorial de la Fundación Universidad de Bogotá Jorge Tadeo Lozano. $1^{\mathrm{a}}$ edición, Santafé de Bogotá, 293 pp.

Rivero, L. H. 1951. Peces larvívoros. Boletín de Historia Natural de la Sociedad Zoológica Felipe Poey, 2 (6): 90-97.

Rodríguez, H. G., G. S. Salazar y J. L. Polo. 1993. Estudio nacional de Colombia. En Diagnóstico sobre el Estado de la Acuicultura en América Latina y el Caribe, Síntesis regional GCP/ RLA/102/ITA. Aquila II. FAO. Doc. de Campo 11: 162-165.

Rosen, D. 1975. A vicariance model of Caribbean biogeography. Syst. Zool., 4: 431-464.

Saikia, S. K y D. N. Das. 2009. Feeding ecology of common carp Cyprinus carpio L. in a rice fish culture system of the Apatani plateau (Arunachal Pradesh, India). Aquatic Ecology, 43 (2): 559-568. 
Unión Internacional para la Conservación de la Naturaleza UICN. 2011. UICN Red List of Threatened Species. UICN, Gland, Switzerland.

Vales, M., A. L. Álvarez, Montes y A. Ávila. 1998. Pisces en: Estudio Nacional sobre la Diversidad Biológica en la República de Cuba. CESYTA, 202-203 pp.

Vergara, R. 1992. Principales características de la ictiofauna dulceacuícola cubana. Editorial Academia, Ciudad de La Habana, 27 pp.

Yalçin, P. K., U. Akyurt y K. Solak. 2001a. Stomach contents of the Catfish Clarias gariepinus (Burchell, 1822) in the river Asi (Turkey). Turkey Journal of Zoology, 25: 461-468.

Yalçin, P. K., U. Akyurt y K. Solak. 2001b. Certain reproductive characteristics of the Catfish Clarias gariepinus (Burchell, 1822) living in the river Asi, Turkey. Turkey Journal of Zoology, 25: 453-460.

Watson, R. E. 1996. Revision of the subgenus Awaous (Chonophorus) (Teleostei: Gobiidae). Ichthyol. Explor. Freshwat., 7 (1): 1-18.

Wilkens, H. 1982. Regressive evolution and phylogenetic age: The history of colonization of freshwaters of Yucatan by fish and Crustacea. Assoc. Mex. Cave Stud. Bull., 8: 237-243.

Zambrano, L y D. Hinojosa. 1999. Direct and indirect effects of carp Cyprinus carpio L. on macrophyte and benthic communities in experimental shallow ponds in central Mexico. Hydrobiologia, 409: 131-138.

[ Recibido: 31 de Julio, 2016. Aceptado para publicación: 18 de septiembre, 2016] 